\title{
Gerencia tecnológica e innovación en la aplicación de variables emprendedoras en las universidades del departamento de Córdoba
}

\section{DEIVI DAVID FUENTES-DORIA ${ }^{a}$}

RESUMEN El objetivo principal del artículo es analizar la gerencia tecnológica y la innovación en la aplicación de variables emprendedoras en instituciones universitarias del departamento de Córdoba. La metodología aplicada tiene un enfoque cualitativo, por tanto, es una investigación de tipo documental descriptiva. Entre los resultados se encontró que las instituciones universitarias no poseen el suficiente manejo de variables emprendedoras que les permita implementarlas en torno a una adecuada gerencia e innovación.

En este sentido, existen bajos niveles de estrategias proactivas enfocadas en los procesos de difusión de conocimiento, lo que apunta a obtener una posición muy baja en los procesos investigativos. Las universidades, a pesar de contar con centros de investigación, no cuentan con los niveles de publicación académica que les permitan mejorar sus procesos investigativos. Por lo tanto, se recomienda implementar estrategias que permita mayores niveles de integración universidad-empresa, para detectar las necesidades del entorno y orientar investigaciones básicas y aplicadas, entorno al mejoramiento de la problemática social.

PALABRAS CLAVE gerencia, tecnología, innovación, variables emprendedoras.

\section{HISTORIA DEL ARTÍCULO}

¿CÓMO CITAR?:

Fuentes-Doria, D. D. (2017).

Gerencia tecnológica e innovación en la aplicación de variables emprendedoras en las universidades del departamento de Córdoba. Perspectiva Empresarial, 4(2), 27-35. http://dx.doi.org/10.16967/rpe. v4n2a3

RECIBIDO: 18 de junio de 2017 APROBADO: 29 de agosto de 2017

CORRESPONDENCIA:

Deivi David Fuentes Doria, Universidad Cooperativa de Colombia, Calle 52a No. 6-79, Barrio La Castellana. Montería - Córdoba, Colombia.

a Doctorante en ciencia-mención gerencia, coordinador de investigación en la Universidad Cooperativa de Colombia y profesor en la Corporación Universitaria CUN.Correo electrónico deividfuentes@hotmail.com. 
¿CÓMO CITO EL ARTÍCULO? HOW TO CITE THIS PAPER?

CHICAGO:

Fuentes-Doria, Deivi David. 2017. "Gerencia tecnológica e innovación en la aplicación de variables emprendedoras en las universidades del departamento de Córdoba". Perspectiva Empresarial 4(2): 27-35. http://dx.doi. org/10.16967/rpe.v4n2a3

MLA:

Fuentes-Doria, Deivi David. "Gerencia tecnológica e innovación en la aplicación de variables emprendedoras en las universidades del departamento de Córdoba". Perspectiva Empresarial 4.2 (2017): 27-35. Digital. http:// dx.doi.org/10.16967/rpe. v4n2a3

\section{Technological management and innovation in the application of entrepreneurial variables to universities in the department of Córdoba}

ABSTRACT The article intends to analyze technological management and innovation in the application of entrepreneurial variables to universities in the department of Córdoba. The applied methodology has a qualitative approach; thus, it is a descriptive documentary research. Some of the results show that universities have not implemented entrepreneurial variables for adequate technology management and innovation.

In this sense, there are low levels of proactive strategies focused on the processes of knowledge dissemination, achieving a very low position in the research processes. Universities, despite having research centers, do not have the levels of academic publishing to improve their research processes. Therefore, it is recommended that they implement strategies that allow higher levels of university-company integration to detect the needs of the environment and to guide basic and applied research in relation to the improvement of social problems.

KEYWORDS management, technology, innovation, entrepreneurial variables.

\section{Gerência tecnológica e inovação na aplicação de variáveis empreendedoras nas universidades do departamento de Córdoba}

RESUMO O objetivo principal do artigo é analisar a gerência tecnológica e a inovação na aplicação de variáveis empreendedoras em instituições universitárias do departamento de Córdoba. A metodologia aplicada tem um enfoque qualitativo, por tanto, é uma investigação de tipo documental descritiva. Entre os resultados constatou-se que as instituições universitárias não possuem o suficiente manejo de variáveis empreendedoras que lhes permita implementá-las em torno a uma gerência e inovação adequadas.

Neste sentido, existem baixos níveis de estratégias proativas enfocadas nos processos de difusão de conhecimento, o que indica obter uma posição muito baixa nos processos de pesquisa. As universidades, apesar de terem centros de pesquisa, não contam com os níveis de publicação acadêmica que as permitam melhorar seus processos de pesquisa. Por isso, é recomendado implementar estratégias que permitam maiores níveis de integração universidade-empresa, para detectar as necessidades do entorno e orientar pesquisas básicas e aplicadas, no âmbito do melhoramento da problemática social.

PALAVRAS CHAVE gerência, inovação, tecnologia, variáveis empreendedoras. 


\section{Introducción}

Las instituciones están experimentado un cambio de cierta importancia en el sistema integrativo de la sociedad actual, con lo cual desplazan los procesos de formación desde los entornos convencionales hacia otros ámbitos. Asimismo, la atención a las demandas generalizadas que los beneficiados reciban, las competencias necesarias para el aprendizaje continuo y la comercialización del conocimiento que genera, de forma simultánea, oportunidades para nuevos mercados y competencias en el sector, entre otros.

En este sentido, el ámbito de aprendizaje varía de forma vertiginosa, pues las instituciones tradicionales, bien sean presenciales, o bien a distancia, deben reajustar sus sistemas de distribución y comunicación (Salinas, 2012). Por tanto, las instituciones pasan de ser el centro de la estrella de comunicación integral, a constituir simples nodos de un entramado de redes entre las que se mueven en unas coordenadas más flexibles, lo cual se ha denominado "ciberespacio".

Por otra parte, los cambios en estas coordenadas espacio-temporales traen consigo la aparición de nuevas organizaciones de enseñanza, las cuales se constituyen como consorcios o redes de instituciones, y cuyos sistemas de enseñanza se caracterizan por la modularidad, así como por la interconexión. Todo esto exige a las instituciones una flexibilización de sus procedimientos y de su estructura administrativa.

Al respecto, Morán \& Myringer (2013) expresan cómo para adaptarse a modalidades de formación alternativas más acordes con las necesidades que esta nueva sociedad presenta, se requiere la existencia de ofertas on-line y de cursos en Internet, o de otro modo los proyectos experimentales de algunos profesores y/o departamentos no presupondrían una operadora social más flexible. Por ello, Bierly, Scott, Tuner \& Lanz (2000) señalan que la aparición del desarrollo de una emergente sociedad de la información como la del conocimiento supone, tanto el análisis, como la evaluación de las necesidades de los usuarios, lo que refleja la sobreoferta de información, del entorno tecnológico y de los recursos disponibles, a fin de que las unidades de información sean capaces de ofrecer un servicio dinámico y adaptado a los requerimientos reales de búsqueda de conocimiento.

Cuando se presentan oportunidades en las que la gestión del conocimiento, la innovación tecnológica y el uso adecuado de las tecnologías de la información y la comunicación (TIC) se manejan de manera conjunta, se logran mejores resultados en términos de generación de patrimonio y de empleo. Son estos los casos en los que se han introducido estos factores a partir de una visión estratégica e integrada, la cual tiene en cuenta los cambios que una innovación tecnológica implica en la organización del trabajo, en la formación del capital humano y en las relaciones de la empresa o de la institución con el exterior.

\section{Fundamentos conceptuales}

El análisis de la gerencia y la innovación en la aplicación de variables emprendedoras desde un marco referencial teórico, permite conocer aquellos elementos que representan su justificación epistémica, para luego abordar de qué manera las mismas se aplican en la realidad organizacional. Esto, ya que, para toda estrategia innovadora, necesariamente, debe transcurrir un lapso que sirva como adaptación a los sujetos que la manipulan, por lo cual es ineludible que se generan una serie de ventajas y desventajas que fortalecen su uso.

Ahora bien, el desarrollo de la gerencia y la innovación en la aplicación de variables emprendedoras ha generado una serie de ventajas en relación con la información, como, por ejemplo, con su acceso. Estos cambios produjeron en las últimas décadas impactos de forma significativa en la estructura social colombiana, cuyo desarrollo se sustenta en las variables emprendedoras evidenciadas, pocas al igual que los avances tecnológicos que ofrecen, tan necesarios para mejorar los estándares de calidad de vida en muchos sentidos. En fin, en cualquier disciplina u ocupación surgen constantemente nuevas formas para asumir la resolución de problemas.

Para Didriksson (2000), las variables emprendedoras son políticas que la operadora social sigue en el desarrollo y el uso de la tecnología. Debido al poder del cambio tecnológico para influir en la estructura del sector industrial y la ventaja competitiva, la estrategia tecnológica es un componente fundamental de la estrategia competitiva de las instituciones.

En este sentido, de acuerdo con Sánchez (2013), el concepto de estrategia tecnológica es más amplio que el de investigación y desarrollo (I+D) tradicional. Comprende no solo la I+D de nuevos productos y procesos, sino también el que 
su acción se deba extender a todas las funciones o subsistemas de la empresa o ámbito universitario en estudio. Por tanto, la estrategia tecnológica es el proceso de adopción y ejecución de decisiones sobre las políticas, las estrategias, los planes y las acciones relacionadas con la creación, la difusión y el uso de la tecnología.

Por otra parte, variables emprendedoras, según Libretti (2009), son aquellas con potencial para un impacto significativo en la empresa durante los próximos tres años desde el momento de su aplicación. Esto, debido a factores tales como el alto potencial disruptivo, la necesidad de inversiones o el riesgo que supondría una adopción tardía; dicho de otro modo, actividades que debería programar cualquier empresa u organización según una planificación estratégica de sus recursos tecnológicos y, cabría añadir, en el contexto actual de la web 2.0, el marketing y la "socialización".

En este marco de ideas, la investigación que se presenta se inclina por la conceptualización que realiza Sánchez (2013), ya que se desarrolla bajo el enfoque de las posibilidades de solución universitarias que se pueden tener con la formulación de variables emprendedoras, además de la presencia continua de la utilización de las tecnologías como medio de gerencia e innovación.

\section{Tipos de variables emprendedoras}

De acuerdo con Sánchez (2012), se encuentran los siguinetes tipos de variables emprendedoras:

- Estrategias competitivas. Hoy en día, de acuerdo con Ferraro (2009), cuando la competencia internacional obliga a las instituciones e industrias a elaborar una estrategia tecnológica que les permita aprovechar todos los posibles beneficios que implica la globalización, las instituciones y empresas que han logrado sobrevivir después de la apertura del mercado se han visto en la necesidad de diseñar nuevas estrategias. De acuerdo con el concepto de estrategia, la estrategia tecnológica debe integrar las metas tecnológicas de las universidades y establecer las acciones a realizar. Porter (2009) señala que la estrategia tecnológica es el enfoque de una operadora social o empresa para el desarrollo y uso de la tecnología. Es un elemento de la estrategia competitiva general, y debe estar en conformidad con las otras actividades de valor. Por otra parte, Sánchez (2012) afirma que las estrategias competitivas representan un papel fundamental en el impulso de la obtención de beneficios de las instituciones universitarias en el sector que quieren determinar, en cuanto alianzas para la producción, tanto de procesos, como de productos, por lo cual estas organizaciones requieren alcanzar estrategias que les proporcionen ventajas. Entre estas estrategias se encuentran:

- Estrategias de diferenciación: consisten en suministrar un servicio único, de manera que el individuo se incline por la selección, ya que esta supera el costo de la diferencia.

- Estrategias de enfoques: consisten en suministrar un servicio a un segmento del mercado más limitado denominado "nicho".

- Estrategias proactivas. Las variables emprendedoras requieren formularse, según Porter (2009), dentro del marco conceptual de la misión y visión de la institución o empresa, con el fin de que cada miembro del equipo de trabajo sepa hacia dónde dirigir los esfuerzos, y se apoye en una política dinámica de investigación y desarrollo cuyo objeto principal sea introducir nuevos servicios, así como procesos hacia el liderazgo exclusivo de sectores. En este sentido, esta estrategia apunta a obtener una posición permanente de liderazgo tecnológico. Este es precisamente un objetivo en el que todos los esfuerzos se concentran para lograrlo, y se inyecta con el mejor presupuesto destinado a la investigación y al desarrollo, así como a la difusión de conocimiento.

- Estrategias reactivas. Las estrategias reactivas se apoyan en mantener el liderazgo competitivo mediante mejoras radicales en procesos y servicios, así como mejoras incrementales en productos que permitan aumentar la facilidad de uso, la calidad y la fiabilidad de los sistemas, a la vez que reducen los costos de producción. Con la utilización de este tipo de estrategias es posible distinguir entre las instituciones que las utilizan y se encuentran en continua innovación, y la que siguen la idea —de acuerdo con Baldwin \& Childs (2009) según la cual vale la pena copiar y distribuir bajo los modelos que presentan una gran capacidad de investigación y desarrollo, aunque existan acuerdos de licencias y know how. 
- Estrategias de baja intensidad. Estas estrategias aprovechan, según Sánchez (2012), huecos que nadie satisface, e intentan por ello encontrar un nicho de mercado poco atractivo para la competencia que ofrezca potencial de crecimiento y rentabilidad aceptables. De acuerdo con Drucker (2012), estas estrategias, según refuerzan la estrategia de enfoque, presentan:

- Estrategia dependiente: la empresa o operadora social acepta el papel subordinado con respecto a las innovaciones de una operadora social o empresa más fuerte, de modo que decide actuar en el mercado sin asumir riesgos tecnológicos y con el objeto de satisfacer una demanda establecida.

\section{Importancia del emprendimiento en las universidades}

Si una universidad produce solo servicios educativos, sus docentes e investigadores podrían desarrollar planes estratégicos únicos que abarquen todas sus actividades de producción investigativa. Estos irían de lo básico utilizado, hasta la innovación de servicios y procesos. Cada uno de estos entes siempre exige una estrategia separada. Como resultado, se distingue entre estrategias a nivel universitario (estratégico), y a nivel de emprendimiento (negocio).

\section{Nivel estratégico (estrategia corporativa)}

Se encuentran en las instituciones universitarias que poseen diversificación, y consiste, según Drucker (2012), en determinar las actuaciones necesarias para obtener una ventaja competitiva mediante la selección y dirección de una combinación de conocimientos. En este sentido, la estrategia conlleva a las iniciativas de administrar los procesos investigativos (proyectos-negocios), así como a apoyar las actualizaciones de los diferentes negocios, capturar sinergias entre centros de investigación y obtener recursos corporativos necesarios y distribuidos entre los negocios.

\section{Nivel de negocio}

Este nivel define, según Sánchez (2000), cómo crear y reforzar la competitividad a largo plazo del negocio. Para ello requiere centrase en: determinar el alcance; responder a los cambios del entorno; que las funciones proporcionen sinergias y apoyen la estrategia de negocio; y desplegar los recursos necesarios para el logro de capacidades.

Para Ansoff (2000), a este nivel se trata de determinar cómo desarrollar lo mejor posible la actividad o las actividades correspondientes a la unidad estratégica, es decir, en un entorno competitivo.

Cuando una organización trabaja en varios negocios diferentes, la planificación puede facilitarse al crear unidades de negocio estratégicas. Una unidad estratégica de negocio (UEN) representa un negocio único o un grupo de negocios relacionados, es decir, es un conjunto de actividades o negocios homogéneos desde un punto de vista estratégico, para el cual es posible formular una estrategia común, y a su vez diferente de la estrategia adecuada para otras actividades y/o unidades estratégicas.

La estrategia de cada unidad es, en sí, autónoma, si bien no independiente de las de las demás unidades estratégicas, puesto que se integran en la estrategia de la empresa. Cada UEN tendrá su propia misión distintiva y competidores diferentes. Esto le permite a la UEN tener una estrategia independiente de los otros negocios de la organización mayor.

\section{Nivel funcional}

De acuerdo con Ansoff (2000), la estrategia a nivel funcional busca responder a la pregunta: ¿Cómo podemos apoyar la estrategia a nivel de negocios? Para las organizaciones que cuentan con departamentos funcionales tradicionales tales como producción, marketing, recursos humanos, investigación y desarrollo, y finanzas, estas estrategias deben apoyar la estrategia a nivel de negocios.

En este tercer y último nivel, la cuestión es cómo utilizar y aplicar los recursos y habilidades dentro de cada área funcional existente en cada actividad o cada unidad estratégica, a fin de maximizar la gerencia e innovación de dichos recursos. Para Sánchez (2000), es el conjunto de actividades que se llevan a cabo con el fin de desplegar los recursos de un departamento concreto. Así, debe apoyar y reforzar la estrategia de negocio para apalancar la ventaja competitiva, e, incluso, requiere complementar y ser coherente con el resto de estrategias funcionales, así como crear sinergias interdepartamentales. 


\section{Gerencia e innovación y la incidencia de los tipos de tecnologías}

En la actualidad, entender cuál es el rol de los tipos de tecnologías de información en torno a la gerencia y la innovación, según Sleight (2000), es la pieza clave para no cometer un error de concepto. Este error radica, algunas veces, en concebir la implantación del conocimiento como una tarea de las tecnologías de la información. Es importante comprender que las tecnologías de la información proveen el marco, pero no el contenido. En este sentido, antes de seleccionar y clasificar las diversas tecnologías de la información para la gerencia y la innovación, es necesario, según Ruggles (2013), entender el significado de la denominadas "herramientas del conocimiento" o software de la administración del conocimiento. Este autor concibe este concepto como el software o la herramienta que apoya y da soporte a las aplicaciones, actividades o acciones tales como la generación, la codificación y la transferencia del conocimiento.

Asimismo, se observa que procesos o actividades de generación tales como el de codificación del conocimiento y selección adecuada de una tecnología de información, pueden ser fundamentales en los objetivos finales -en este caso de investigación-, por ser la forma de evidenciar su importancia en el contexto universitario colombiano.

En el mismo contexto, el proceso de selección de las tecnologías de la información tiene por objeto encontrar aquellas que integren y cubran de forma más específica los puntos focales de la gerencia y la innovación. Para esto se toman en cuenta dos bases importantes: 1. Existe un mercado con una gran cantidad de tecnologías de la información de diversas características, por tanto, la clasificación está sujeta a la identificación de aspectos que generen valor en los modelos de creación, como, por ejemplo, el de gerencia e innovación: y 2. Se requiere tomar en cuenta clasificaciones en investigaciones y artículos de anteriores autores como antecedentes que avalen el estudio.

Así, entonces, con el propósito de clasificar los tipos de tecnologías inmersas en la difusión de conocimiento, se encuentran diferentes posiciones. Para Jiménez (2011), por ejemplo, existen tecnologías según su diferenciación en el uso y los resultados que arrojan. A continuación, se describen algunos tipos de estas tecnologías:

- Tecnologías claves. Hacen referencia a los grandes descubrimientos científicos que han aparecido de manera irregular, e inclusive, sorpresivamente, a lo largo de la historia, y que han facilitado el quehacer del ser humano (Jiménez, 2011). Entre estas se pueden mencionar la energía eléctrica, la máquina a vapor, el motor de explosión, el petróleo, microprocesadores $y$, entre lo más reciente, la nanotecnología. Esto ha llevado a que en la actualidad se encuentre presente la informática, las telecomunicaciones, la robótica, nuevos materiales de fabricación, el láser y la biotecnología, entre otros. Esto se ha convertido en una especie de emergencia que ha revolucionado todas las actividades, tanto económicas, como sociales.

- Tecnologías alternativas. La mayor parte de las tecnologías alternativas, según Jiménez (2011), han nacido de las críticas formuladas en contra de los efectos indeseables del sistema industrial (la contaminación, el despilfarro y los problemas de salud de los trabajadores, entre otros). Por otra parte, para Ruiz (2009), las tecnologías de difusión del conocimiento van más allá del simple uso del Internet; estas requieren de un conocimiento para su utilización en las áreas que se desean desarrollar, bajo la fluidez de formas tecnológicas de difusión, por lo cual las divide en:

- Tecnología blanda: el término engloba a los conocimientos de planificación, administración y comercialización, y deja de lado el saber técnico al respecto. Se denomina "blanda" ya que hace referencia a información no tangible, en contraposición con la tecnología dura, que sí es tangible.

- Tecnología dura: término que se utiliza para designar a los saberes exclusivamente técnicos, aplicados a la producción de maquinarias, productos y materiales, entre otros.

Ferreros (2009) cataloga las tecnologías de difusión de conocimiento de igual manera en tecnologías blandas, enfatizando que son aquellas en las que su producto no es objeto tangible, y pretenden mejorar el funcionamiento de las instituciones u organizaciones para el cumplimiento de sus objetivos. Dichas organizaciones pueden ser empresas industriales, comerciales o de servicios -o bien instituciones integrales-, con o sin fines de lucro. Al respecto, el mismo autor señala que entre las ramas de la tecnología blandas se destacan la educación (en lo que respecta al proceso de enseñanza), la organización, la administración y la 
contabilidad, así como las operaciones, la logística de producción, el marketing, la estadística y la psicología de las relaciones humanas.

\section{Innovación en los procesos de gerencia tecnológica}

De acuerdo con Cuervo (2009), se debe estudiar, básicamente, la evolución del entorno. Se requiere de la búsqueda de sectores en los cuales se presentan oportunidades, así como analizar el estado de las tecnologías y aquellas que se prevén como sustitutas. A su vez, es necesario buscar nuevas tecnologías que aumenten la rentabilidad. Finalmente, es muy importante mantener una estrecha relación entre la estrategia tecnológica y la de toda la empresa. Al momento de realizar un estudio para establecer una estrategia tecnológica, se deben valorar dos visiones diferenciadas:

- Análisis externo. Se trata de analizar cuál es el estado del arte en ese momento. Esto se puede llevar a cabo mediante la información proveniente de las reglas internacionales de comercio y de las patentes, las tendencias del mercado nacional e internacional de bienes, y de las tendencias científicas y tecnológicas. De tal manera, consiste en evaluar las oportunidades y las amenazas existentes. Aquí juega un papel importante la vigilancia tecnológica.

\section{Metodología}

La metodología aplicada para la determinación del tipo de investigación, así como para como su diseño, contiene también la sistematización desde el orden científico. Es decir, se describen las técnicas e instrumentos aplicados para la medición del comportamiento de las variables, la delimitación de la población, las técnicas e instrumentos de recolección de datos, la validez y la confiabilidad del instrumento, el tratamiento estadístico de los datos y el procedimiento de la investigación.
El propósito de este estudio es analizar la gerencia y la innovación en la aplicación de variables emprendedoras, y con ello indagar en las creaciones existentes, en diferentes áreas, que apoyen a las comunidades universitarias. Del mismo modo, dadas sus características, se considera de carácter descriptivo, según Tamayo \& Tamayo (2000), debido a que el estudio se orienta a recolectar información relacionada con el estado real de las personas y de los fenómenos, tal cual como se presentan en el contexto de las instituciones del departamento de Córdoba. Por lo tanto, la investigación se enmarcó dentro de una investigación de tipo descriptiva.

En este sentido, Hernández, Fernández \& Baptista (2006) señalan cómo la investigación que busca especificar las propiedades importantes de personas, grupos, comunidades o cualquier otro fenómeno que sea sometido a análisis, es una investigación descriptiva. Según estos autores, en un estudio descriptivo se seleccionan una serie de cuestiones y se mide cada una de ellas de forma independiente, con el fin de describir lo que se investiga.

El término diseño se refiere al plan o materia concebida para obtener información que se desea. Albert (2006) plantea que el diseño se define como el plan o las estrategias que se desarrollan con el propósito de obtener la información que se requiere en una investigación. Según Pérez (1997), el diseño de la investigación es un esquema general del trabajo, necesario para comprobar la hipótesis o, en general, medir variables.

El diseño de la investigación que se presenta es de tipo no experimental, según Hernández et al. (2006), ya que se basa en observar situaciones o fenómenos existentes, los cuales no han sido provocados ni manipulados intencionalmente por el investigador. Así, un estudio no experimental es aquel que se realiza sin manipular deliberadamente variables o asignar de manera aleatoria a los sujetos o las condiciones (2006).

Por otra parte, las universidades objeto de estudio del departamento de Córdoba fueron: Universidad Cooperativa de Colombia,

TABLA 1. Estrategias competitivas

\begin{tabular}{lcccc}
\hline ACTIVIDAD & $\begin{array}{l}\text { UNIVERSIDAD } \\
\text { COOPERATIVA }\end{array}$ & $\begin{array}{l}\text { UNIVERSIDAD DEL } \\
\text { SINÚ }\end{array}$ & $\begin{array}{l}\text { UNIVERSIDAD } \\
\text { CUN }\end{array}$ & $\begin{array}{l}\text { UNIVERSIDAD } \\
\text { CECAR }\end{array}$ \\
\hline $\begin{array}{l}\text { Nuevos programas académicos ajustados } \\
\text { a la necesidad del entorno }\end{array}$ & Alto & Alto & \multirow{2}{*}{ Bajo } & Bajo \\
\hline
\end{tabular}


Universidad del Sinú, Corporación Universitaria del caribe (CECAR), y Corporación Unificada de educación Superior (CUN).

\section{Resultados}

En las universidades del departamento de Córdoba existen algunas fortalezas relacionadas con el tipo de estrategia de baja y media intensidad en las coordinaciones objetos de estudio en esta investigación. Por lo cual son de gran importancia para el enfoque y el criterio establecido.

Es posible argumentar que las estrategias de competitividad son las menos utilizadas por las universidades estudiadas, aunque existen algunas con debilidades en los procesos de oferta académica. Al respecto, la utilización de este tipo de estrategias se distingue de las que las instituciones utilizan para mejorar sus procesos académicos y aportes a la región.

De igual forma, el nivel estratégico lo utilizan las coordinaciones universitarias para las iniciativas de programas académicos que puedan aportar soluciones a problemáticas de la región, apoyar las actualizaciones de los diferentes negocios, capturar sinergias entre centros de investigación y las organizaciones, así como obtener recursos corporativos necesarios y distribuidos entre los negocios.

Dados los resultados presentados, existe un nivel bajo en las ofertas de programas virtuales de las universidades del departamento de Córdoba, debilidad que afecta a todas las personas que, por ocupación, no cuentan con disponibilidad para asistir a programa presenciales.
En este sentido, existen bajos niveles de estrategias proactivas enfocadas a los procesos de difusión de conocimiento, lo que apunta a obtener una posición muy baja en los procesos investigativos. Las universidades, a pesar de contar con centros de investigación, no cuentan con los niveles de publicación académica que les permitan mejorar sus procesos investigativos.

\section{Conclusiones}

Se concluye de la investigación que el tipo de tecnología más utilizada en las universidades es la dura, por tanto, en las coordinaciones de estudios de las instituciones de Córdoba, Colombia, no le dan suficiente valor a las tecnologías, desconociendo el impacto positivo que generan en el entorno organizacional para el mejoramiento de las condiciones sociales, culturales y educativas. La investigación se inclina por los tipos de tecnologías para la difusión de conocimientos con bajos niveles. En tal sentido, este debe adaptarse y readaptarse a las necesidades requeridas en el mercado laboral, de manera que deben garantizarse acciones que faciliten la formación de sistemas curriculares aptos para el desarrollo, tanto de la gestión educativa, como de la práctica empresarial. Por medio de la relación analizar-sintetizar, los procesos de tecnología e innovación, o bien el equipo de gestión, pueden desarrollar una educación de calidad a condición de incentivar de manera permanente las funciones de investigación, así como de análisis de la realidad particular y singular en el que opera el sistema educativo y cada una de las instituciones.

TABLA 2. Estrategias tecnológicas

\begin{tabular}{lccccc}
\hline ACTIVIDAD & $\begin{array}{l}\text { UNIVERSIDAD } \\
\text { COOPERATIVA }\end{array}$ & \multicolumn{2}{l}{$\begin{array}{l}\text { UNIVERSIDAD DEL } \\
\text { SINÚ }\end{array}$} & $\begin{array}{l}\text { UNIVERSIDAD } \\
\text { CUN }\end{array}$ & $\begin{array}{l}\text { UNIVERSIDAD } \\
\text { CECAR }\end{array}$ \\
\hline Programa ofertado de manera virtual & Bajo & Bajo & Bajo & Alto \\
\hline Fuente. elaboración propia. & & & & &
\end{tabular}

TABLA 3. Estrategias tecnológicas

\begin{tabular}{lcccc}
\hline ACTIVIDAD & $\begin{array}{l}\text { UNIVERSIDAD } \\
\text { COOPERATIVA }\end{array}$ & $\begin{array}{l}\text { UNIVERSIDAD DEL } \\
\text { SINÚ }\end{array}$ & $\begin{array}{l}\text { UNIVERSIDAD } \\
\text { CUN }\end{array}$ & $\begin{array}{l}\text { UNIVERSIDAD } \\
\text { CECAR }\end{array}$ \\
\hline $\begin{array}{l}\text { Procesos de difusión de conocimiento } \\
\text { (Revistas científicas) }\end{array}$ & Bajo & Bajo & Bajo & Alto \\
\hline
\end{tabular}


Por otra parte, las estrategias proactivas desarrolladas por las instituciones universitarias son muy bajas, pues presentan procesos que requieren de un análisis y una síntesis orientados por una intervención de mejoramiento de la gestión investigativa. El análisis como etapa del diagnóstico requiere llegar a una síntesis, a la reconstrucción de la realidad bajo un esquema, modelo o analogía, esto es, todos los instrumentos conceptuales que posibiliten luego diseñar alternativas de intervención. Asimismo, contar con herramientas para el abordaje de los procesos de cambio e innovación, y conocer cuál debe ser la información, lo que le permita anticiparse a cambios y crisis.

En fin, las instituciones no poseen suficiente manejo de variables emprendedoras para implementar en la gerencia y la innovación de cada facultad o escuela, lo que demuestra poco interés en la utilización de estrategias tecnológicas e investigativas.

\section{REFERENCIAS}

Albert, T. (2006). El proyecto de investigación. México: Editorial Limusa.

Ansoff, M. (2000). Modelos de planeación estratégica de negocio. Madrid: MacGraw Hill.

Baldwin, T., \& Childs, U. (2009). Universal neonatal hearing screening moving from evidence to practice. Nueva York: Macmillan.

Bierly, N., Scott, H., Tuner, H., \& Lanz, J. (2000). El contexto histórico de la evolución de las tecnologías de información. Madrid: Editorial Crítica.

Cuervo, J. (2009). Tecnologías disruptivas en el ámbito integrativo universitario. Madrid: MacGraw Hill.

Didriksson, A. (2000). La operadora social de la innovación, una estrategia de transformación para la construcción de instituciones del futuro. Caracas: Unesco.
Drucker, P. (2012). Gerencia para el futuro. Colombia: Grupo Editorial Norma.

Ferraro, N. (2009). El uso de nuevas formas de comunicación integral. Editorial Caracas: Limusa.

Ferreros, J. (2009). Las corrientes filosóficas y la justificación de los procesos. Buenos Aires: Editorial El Lago.

Hernández, R., Fernández, C., \& Baptista, P. (2006). Metodología de la investigación. México: McGraw-Hill Interamericano, S. A.

Jiménez, A. (2011). Tecnología y ciencia en la producción de competitividad. New Jersey: Prince Hall.

Libretti, V. (2009). Un modelo de unidad de vinculación operadora social-empresa. FUNDES-USB. Revista Espacios, 20.

Morán, L., \& Myringer, B. (2013). Flexible learning and university change. En K. Harry (Ed.), Higher education through open and distance learning, (pp. 57-72). Londres: Routledge.

Pérez, H. (1997). Metodología de la investigación. Maracaibo: Editorial de la Operadora Social del Zulia.

Porter, A. (2009). The technological dimension of competitive strategy. Nueva York: JAI Press.

Ruggles, R. (2013). Knowledge Management Tools. Oxford: Butterworth-Heinemann.

Ruiz, L. (2009). Tecnologías según el uso. Madrid: MacGraw Hill.

Salinas, A. (2012). Innovación docente y uso de las TIC en la enseñanza universitaria. Revista de Operadora Social y Sociedad del Conocimiento (RUSC). 1(1), 1-16.

Sánchez, J. (2000). El uso progresivo de los avances.

Sánchez, J. (2012). La educación y el proceso de formación tecnológica.

Sánchez, E. (2013). Estrategias de innovación. Madrid: Thomson Editores.

Sleight, W. (2000). Las tecnologías de la información. México: Editorial Grijalbo.

Tamayo, R., \& Tamayo, M. (2000). El proceso de la investigación científica. México: Limusa, Noriega Editores. 